\title{
大兴安岭北部地区原始林白桦种群 的世代结构
}

\section{邱 扬 ${ }^{1}$ 李湛东 ${ }^{2}$ 张玉钧 $^{2}$ 徐化成 $^{2}$ 于汝元 ${ }^{2}$ 张希来 $^{3}$}

（1 北京师范大学地理学与遥感科学学院, 北京 100875) (2 北京林业大学, 北京 100083) (3 陕西省国土资源厅土地整理中心, 西安 710045)

摘 要 在景观火干扰历史重建的基础上, 该文研究了大兴安岭北部地区原始林白桦(Betula platyphylla)种群的世 代结构及其与火干扰、立地条件之间的关系。结果表明 :白桦种群基于世代数的世代结构类型丰富，从一代型到五 代型都有, 空间分布呈斑块镶嵌状。以一代型与二代型为主要类型, 空间分布集中, 其它类型的世代结构空间异质 性较高。世代数受火干扰频率、火干扰强度及两者的综合影响,相对来说以火干扰频率的影响更为显著。低频或 高强类的火干扰易造成种群的世代数减少; 高频或低强类的火干扰易造成世代数增多。世代数还随林型组类型而 变化，以溪旁林组 > 赤杨 (Alnus mandshurica) 林组 > 杜香 ( Ledum palustre) 林组。

白桦种群基于火干扰的世代结构类型也很丰富, 存在 3 大类、 5 亚类和 11 小类, 空间分布呈斑块镶嵌状。其中 以火后优势火前劣势型和更新优势火前劣势型为主要类型, 空间分布较聚集, 其它类型的空间异质性较高。这种 世代结构也受火干扰频率、火干扰强度及两者的综合影响。随着火频增高或者火强减弱,白桦种群的自我恢复性 和抗火性增强, 火后恢复性减弱。中高强火烧地上,世代结构为火后优势火前劣势型, 低强火烧地上为更新优势火 前劣势型。世代结构还随立地条件而变化。不同林型组之间,白桦种群的优势世代(火后代和更新代)存在显著的 数量差异, 但是世代结构保持为火后优势火前劣势型, 偏东坡为火后优势火前劣势型, 偏西坡及北坡为更新优势火 后劣势型 ,224 林班为火后优势型 218 林班却为更新优势型。

关键词 大兴安岭 原始林 白桦种群 世代结构 火干扰 立地

\section{STUDY ON COHORT STRUCTURE OF BETULA PLATYPHYLLA POPULATION IN VIRGIN FOREST , NORTHERN REGION OF DAXING' ANLING MOUNTAINS , CHINA}

\author{
QIU Yang ${ }^{1}$ LI Zhan-Dong ${ }^{2}$ ZHANG Yu-Jun ${ }^{2} \quad$ XU Hua-Cheng ${ }^{2} \quad$ YU Ru-Yuan ${ }^{2}$ and ZHANG Xi-Lai ${ }^{3}$ \\ (1 School of Geography, Beijing Normal University, Beijing 100875 , China) (2 Beijing Forestry University , Beijing 100083 , China) \\ (3 Land Consolidation and Rehabilitation Center, Department of Land and Resource of Shaanxi Province , Xi' an 710045 , China)
}

\begin{abstract}
Background and Aims Natural fire is a frequent driving force of communities and populations. However, the cohort structure of Betula platyphylla in the virgin forest of the northern region of Daxing' anling Mountains , China, has rarely been studied. The aim of this paper is to explore the abundance, type and spatial distribution of the cohort structure of $B$. platyphylla , based on the number of cohorts , the fire regime and their relationship to site factors.
\end{abstract}

Methods We used an integrative approach to statistically analyze natural fire disturbance and population structure of $B$. platyphylla. Data on fire disturbance and population structure were collected from 96 plots distributed throughout the landscape. Fire history at the landscape scale was reconstructed based on tree regeneration and fire scars. Population cohort structure was recognized based on the number and regeneration type of cohorts identified.

Key Results Cohort structure based on the number of cohorts is diverse , consisting of one through five cohort groups with a patch-mosaic distribution in the landscape. The main type , i. e. , groups with one and two cohorts are aggregated in space, while the others have a heterogeneous distribution. The number of cohorts is influenced by fire frequency, fire severity and their interaction , but the effect of fire frequency is greatest. Fires of low frequency or high severity increase the number of population cohorts , while fires of high frequency or low 
severity decrease the number of cohorts. In addition, the number of cohorts differs by forest type, with the number of cohorts decreasing from the forest type near streams to the type with Alnus mandshurica to the type with Ledum palustre. Cohort structure based on regeneration type is also diverse, with three groups, five subgroups and 11 types distributed in patch-mosaic. The main sub-types, i.e. , post-fire cohort dominance and pre-fire cohort inferiority and regeneration cohort dominance and pre-fire cohort inferiority , are distributed aggregately, while the others are spatially heterogeneous. Cohort structure is strongly affected by fire frequency, fire severity and their interaction. With increasing of frequency and decreasing of severity , the relative importance of both the regeneration cohort and the pre-fire cohort increases while that of the post-fire cohort decreases. The sub-group of post-fire cohort dominance and pre-fire cohort inferiority occurs on sites with mid- and high-severity fires, while the sub-group of regeneration cohort dominance and pre-fire cohort inferiority occurs on sites with low-severity fires. Both the group and the sub-group of cohort structure change with different types of sites. The cohort structure type is the same for sub-group of post-fire cohort dominance and pre-fire cohort inferiority on all sites, although there is significant difference in the relative importance of the dominance cohorts (i.e. , post-fire and regeneration cohorts) among different types of sites. The sub-group of post-fire cohort dominance and pre-fire cohort inferiority is found on sites toward the east, while the sub-group of regeneration cohort dominance and pre-fire cohort! inferiority is on sites toward the west and north. The group of post-fire cohort is located on forest compartment 224 , while the group of regeneration cohort dominates on forest compartment 218 .

Conclusions This study suggests that the cohort structure of $B$. platyphylla is highly diverse, with several dominant types of cohorts. B. platyphylla is weak in resistance to natural fires, but can maintain its role in the landscape after fire by strong resilience.

Key words Daxing' anling Mountains , Virgin forest , Population , Betula platyphylla , Cohort structure , Fire disturbance , Site

干扰已成为当代生态学研究的活跃领域。干扰 被认为是生态系统的正常行为, 是群落发展的驱动 力 甚至是种群维持的机制 (Pickett \& White, 1985 ; Oliver \& Larson , 1990 ; Glin-Leven et al. , 1992 ; 徐化 成等, 1997)。许多学者结合干扰研究种群年龄结 构 提出了年龄波” (Age wave)或 世代” (Cohort) 的 概念, 并为此开展了一些种群年龄结构与干扰关系 的研究 (Oliver \& Larson, 1990 ; 范兆飞等, 1992 ; 徐 化成等, 1993 ; 邱扬和杜建林, 1997 ; 杨允菲等, 2001 ; 孙晓玉等，2005)。

大兴安岭北部地区是我国重要林区,也是林火 多发区。火干扰是该区生态系统的重要因子, 对该 区植被的形成与维持起着举足轻重的作用(顾云春， 1985 ; 郑焕能等，1986; 徐化成等，1997)。近年来， 有关兴安落叶松 (Larix gmelinii) 种群年龄结构研究 较多(赵惠勋等，1987; 孟宪宇，1989; 范兆飞等， $1991,1992)$ 邱扬等 $(1997,2003)$ 研究了兴安落叶松 种群的世代结构和稳定性及其与火干扰和立地条件 的关系。相对来说, 白桦 (Betula platyphylla) 种群年 龄结构的研究相对薄弱。邱扬等 (1998)研究了白桦 种群的稳定性及其与自然火干扰的关系。在这些基
础上, 本文以景观尺度上火干扰历史研究 (徐化成 等，1997)为基础, 进一步研究了白桦种群的世代结 构及其与火干扰、立地条件之间的关系。

\section{1 研究地区}

研究地点位于内蒙古大兴安岭林管局阿龙山林 业局南娘河林场的一个集水区(即 224 林班和 218 林班)。该局地处大兴安岭北部, $121^{\circ} 6^{\prime} 29^{\prime \prime}$ $122^{\circ} 45^{\prime}$ E $51^{\circ} 33^{\prime} \sim 52^{\circ} 33^{\prime} \mathrm{N}$ 。该地气候寒冷, 少雨。 年均温 $-5{ }^{\circ} \mathrm{C}$, 绝对最高气温为 $35{ }^{\circ} \mathrm{C}$, 最低气温 $-49.6{ }^{\circ} \mathrm{C}$ 。年均降水量 $400 \sim 500 \mathrm{~mm}$, 多集中在 $7 \sim$ 8 月。年均相对湿度为 $70.9 \%$, 无霜期仅 $65 \sim 75 \mathrm{~d}$ 。 地带性土壤为漂灰土。地带性植被为兴安落叶松 林。春秋两季极易发生火烧。

研究地点面积 $542.605 \mathrm{hm}^{2}$, 海拨在 $760 \sim 1100$ $\mathrm{m}$ 之间。坡向多为阴坡或半阴坡, 坡度多小于 $15^{\circ}$ 。

\section{2 研究方法}

本研究采用景观尺度上半随机地布设 $4 \mathrm{~m} \times 50$ $\mathrm{m}$ 的带状样地，利用现存死活物重建火史以及种群 划分世代的方法, 详细方法参看文献 ${ }^{1)}$ (徐化成等， 
1997 ; 兵扬等，1997，1998，2003)。

2.1 样地布设

1993 年,在研究区内, 布设南北间距为 $200 \mathrm{~m}$, 呈东西走向的样带共 14 条。以两个林班交界线为 起点,在 224 林班内每一条样带上每 $200 \mathrm{~m}$ 布设一 个样地, 在 218 林班内, 因林相简单, 每隔一条样带 上每 $400 \mathrm{~m}$ 布设一个样地。如遇有林相变化, 则加 设样地。样地总数 96 个。

\section{2 样地调查}

首先调查能发现的全部火疤木, 记载火疤木的 坐标、火烧年代、火疤高度、年龄以及一般环境特征。 火烧年代的确定采用查数形成层至木炭层的年轮的 方法 ${ }^{1)}$ (徐化成等，1997），3 次正负误差在一年内。 然后调查样地的一般环境状况、林冠结构, 记载样地 内树高大于 $3 \mathrm{~m}$ 的活立木的年龄和径级、树高小于 $3 \mathrm{~m}$ 的幼树年龄。最后调查粗木质残体状况 (包括 枯立和倒木)。

\section{3 火干扰状况的确定}

以火疤木的火烧年代为标准，结合样地的年龄 结构和林冠层结构综合判别每个样地的火烧次数及 年代。研究发现，从 1825 年至今共发生了 14 次火 烧, 最近一次火烧为 1938 年 ${ }^{1)}$ (徐化成等, 1997)。 根据每个样地的火烧次数,采用最小距离逐步聚类 法把每个样地的火频分为 3 类: 低频类 (火烧次数 $\leqslant$ 2 次)、中频类 (3 次 $\leqslant$ 火烧次数 $\leqslant 5$ 次) 和高频类 (火 烧次数 $\geqslant 6$ 次)。

利用火前残存树的数量、粗木质残体状况、火后 更新状况 结合火疤或熏黑高度、成疤时的树龄, 综 合确定 5 个级别的火烧强度得分 (极弱 1 分、弱度 2 分、中等 3 分、强度 4 分、极强 5 分)。在此基础上, 采用最小距离逐步聚类法把每个样地的火强也分为 3 类: 低强类 (火强得分 $\leqslant 2.4$ 分)、中强类 $(2.5$ 分 $\leqslant$ 火强得分 $\leqslant 3.6$ 分) 和高强类 ( $\geqslant 3.7$ 分)。

综合火频分类与火强分类的组合，把所有 96 个
样地的火干扰分为 9 类。研究发现, 除了高频高强 类之外, 其它 8 类都存在。其中, 中频低强类所占面 积最大 $(33.6 \%)$, 高频低强类 $(17.5 \%)$ 与中频中强 类 $(17.2 \%)$ 所占面积中等, 其它 5 类所占面积都较 小1)(徐化成等，1997)。

\section{3 结果与分析}

\section{1 基于世代数的世代结构}

3.1.1世代的划分、世代结构类型及其空间分布 森林火作为一种重要的自然干扰因素, 其对林 木种群年龄结构的影响，国外已有诸多报道 (Oliver \& Larson, 1990 ; Pickett \& White, 1985)。森林火可 促进白桦的更新(邱扬等，1998；单建平等，1990)， 其年龄波的形成与森林火具有密切关系(范兆飞等， 1992)。基于这些理论与现象 根据每个样地上的每 次火烧年代与每株树木的年龄，本研究把每次火干 扰后新补充的全部林木植株定义为一个世代 (Oliver \& Larson，1990) 林冠下天然更新(即未遭受火干扰 而且树高小于 $3 \mathrm{~m}$ 的幼苗幼树) 列为一个世代。可 见，每一个世代相当于一个年龄波。因而，按世代数 的多少, 可把白桦种群的世代结构划分为一代型、二 代型、三代型、…

从表 1 可见，除了少数地块没有白桦分布外，白 桦种群的世代结构较丰富，从一代型到五代型都存 在。但是世代结构以一代型与二代型为主, 世代数 过多 (如四代型和五代型)的世代结构都较少。一代 型与二代型的面积分别为 149.15 和 $242.39 \mathrm{hm}^{2}$, 占 整个研究区的 $27.5 \%$ 和 $44.7 \%$,显然两者面积之和 超过景观的 $50 \%$,为景观世代结构的本底。这两种 世代结构的空间分布较集中, 以一到两个主斑块及 众多小斑块组成。一代型主要分布在 224 林班中下 部:二代型的主要斑块广泛分布在 224 林班中上部 及 218 林班中下部。其它世代结构类型不仅数量较 少,而且其斑块较破碎 ,空间异质性较高。

表 1 白桦种群世代结构的面积分布

Table 1 Area distribution of the cohort structure of Betula platyphylla population

\begin{tabular}{|c|c|c|c|c|c|c|c|}
\hline $\begin{array}{c}\text { 世代结构 } \\
\text { Cohort Structure }\end{array}$ & 零代型 & 一代型 & 二代型 & 三代型 & 四代型 & 五代型 & 总计 \\
\hline $\begin{array}{l}\text { 面 积 Area } \\
\left(\mathrm{hm}^{2}\right)\end{array}$ & 27.64 & 149.15 & 242.39 & 70.49 & 40.30 & 12.65 & 542.61 \\
\hline 百分率 Percent & $(\%)$ & 27.5 & 44.7 & 13.0 & 7.4 & 2.3 & 100.0 \\
\hline
\end{tabular}




\subsection{2 世代数与火干扰的关系}

采用线性回归法来探讨火干扰频率 (火干扰次 数) 和平均火干扰强度这两个单因素与种群世代数 的关系。

\subsubsection{1 火干扰频率对世代结构的影响}

火干扰次数 $(x)$ 与白华种群的世代数 $(y)$ 的线 性回归式如下 :

$$
y=0.66+0.34 x \quad n=91, r=0.62, \alpha=0.001
$$

可见, 火干扰频率对白桦种群世代数的影响极 端显著。随火干扰次数的增多, 白桦种群的世代数 显著增多，即高频度火后易造成种群的世代数增多， 而低频度火干扰后易形成世代数较少的世代结构。

3.1.2.2 火干扰强度对世代结构的影响

平均火干扰强度 $(x)$ 与白桦种群的世代数 $(y)$ 的线性关系如下式 :

$$
y=3.20-0.42 x \quad n=91, r=0.37, \alpha=0.001
$$

火干扰强度对白桦种群的世代结构的影响也很 显著。世代数随火干扰强度的增大而减少。即高强 度的火干扰易造成种群的世代数减少, 而低强度的 火干扰之后易形成多代的种群结构。

\subsubsection{3 火干扰频率与火干扰强度的综合影响}

采用方差分析与多重比较法,分析世代数在 8 种火干扰类型之间的差异, 探讨火干扰次数与火干 扰强度对世代数的综合作用。

方差分析表明，不同火干扰类型间白桦种群的 世代数有显著差异。多重比较结果表明(表 2)，3 类 火频之间 (即低频类、中频类和高频类) 都存在显著 差异，但是火频相同而火强不同类型之间(如中频低 强类、中频中强类、中频高强类) 都没有显著差异。 可见，火干扰频率对世代数的影响比火干扰强度显 著(表 2)。随着火干扰次数的增加,白桦种群世代 数显著增加, 其中低频类都为一代型, 中频类为二代 型，高频类为三代型。相反，火频相同而火强增高， 世代数变化不显著,世代结构类型基本一致。

\section{1 .3 世代数与立地条件的关系}

经数量化模型 III 处理发现, 在海拨、坡向、坡度、 坡位与林型组等立地因子中，只有林型组对世代数有 显著影响。采用方差分析与多重比较的方法来讨论 白桦种群的世代结构在不同林型组之间的变化。

方差分析表明 ,白桦种群的世代数在不同林型 组之间存在显著差异。由表 3 可见, 白桦种群的世 代数以溪旁林组为最多 (2.5 代) , 赤杨 (Alnus mandshurica) 林组居中 (2.1 代), 杜香 (Ledum palustre) 林 组 (1.6 代)世代数最少。

表 2 不同火干扰类型的白桦种群的世代结构类型与世代数

Table 2 Sub-group of cohort structure and number of cohort of Betula platyphylla population among different types of fire disturbance

\begin{tabular}{llc}
\hline \multicolumn{1}{c}{$\begin{array}{c}\text { 火干扰类型 } \\
\text { Type of fire disturbance }\end{array}$} & $\begin{array}{c}\text { 世代结构 } \\
\text { Cohort structure }\end{array}$ & $\begin{array}{c}\text { 世代数 } \\
\text { Number of cohort }\end{array}$ \\
\hline 低频低强类 Low frequency \& low severity & 一代型 1-cohort & $1.50^{\mathrm{ABC}}$ \\
低频中强类 Low frequency \& middle severity & 一代型 1-cohort & $0.93^{\mathrm{A}}$ \\
低频高强类 Low frequency \& high severity & 一代型 1-cohort & $1.25^{\mathrm{A}}$ \\
中频低强类 Middle frequency \& low severity & 二代型 2-cohort & $2.09^{\mathrm{BC}}$ \\
中频中强类 Middle frequency \& middle severity & 二代型 2-cohort & $1.80^{\mathrm{B}}$ \\
中频高强类 Middle frequency \& high severity & 二代型 2-cohort & $1.91^{\mathrm{BD}}$ \\
高频低强类 High frequency \& low severity & 三代型 3-cohort & $2.75^{\mathrm{CDE}}$ \\
高频中强类 High frequency \& middle severity & 三代型 3-cohort & $2.88^{\mathrm{BE}}$ \\
\hline
\end{tabular}

表中字母表示不同火干扰类型之间多重比较结果。两两之间若有一个字母相同则表示差异不显著 $(p>95 \%)$ The letters represent the results from multiple comparison between different fire types. The same letters show the insignificant difference between two types $(p>95 \%)$

表 3 不同林型组之间白桦种群的世代数

Table 3 Number of cohort of Betula platyphyllai population among forest type groups

\begin{tabular}{ccccc}
\hline $\begin{array}{c}\text { 林型组 } \\
\text { Forest type group }\end{array}$ & $\begin{array}{c}\text { 溪旁林组 } \\
\text { Group near streams }\end{array}$ & $\begin{array}{c}\text { 赤杨林组 } \\
\text { Group of Alnus mandshurica }\end{array}$ & $\begin{array}{c}\text { 杜香林组 } \\
\text { Group of Ledum palustre }\end{array}$ & $\begin{array}{c}\text { 平均 } \\
\text { Mean }\end{array}$ \\
\hline 世代数 Number of cohort & 2.5 & 2.1 & 1.6 & 2.0 \\
\hline
\end{tabular}




\section{2 基于火干扰的世代结构}

\subsection{1 白桦种群世代的划分与世代结构类型}

前一节是以世代数为标准来划分世代结构类型 的。本节将从干扰与种群更新的角度, 根据每个样 地每次火烧年代和每株植株的年龄，以最后一次火 干扰为标准, 把种群划分为 3 个世代: : $)$ 火前代:指 最近一次火干扰之后的残存林木, 反映种群的抗火 性以及在景观中的地位 2) 火后代 指最近一次火干 扰之后更新起来的未遭受火烧而且树高 $>3 \mathrm{~m}$ 的活 立木，反映种群的火后恢复力；3) 更新代: 指林冠下 天然更新的未遭受火烧而且树高 $\leqslant 3 \mathrm{~m}$ 的幼苗幼 树，反映种群的自我恢复力 (邱扬等，1997，1998， 2003)。

采用一个相对指标(即世代相对值)来代表 3 个 世代的对比关系。世代相对值是指某世代的单位面 积的株数在种群的单位面积的总株数中所占的百分 率。按 3 个世代的数量对比关系 (即世代相对值) 来 把兴安落叶松种群的世代结构类型划分为更新优势 型、火后优势型和火前优势型 3 大类、9 亚类,再进 一步细分成 15 种小类。可见，这种世代结构类型的 划分可反映种群的抗火性、火后恢复性和自我恢复 性这 3 种特性的综合对比关系及维持其在景观中的 地位的机制(兵扬等，1997，1998，2003)。

更新优势型指更新代存在并且数量最多。根据 火后代与火前代的存在与否及其数量关系又可分为 3 种亚型: 1) 更新独优型:火后代与火前代这两个世 代不存在, 只有更新代, 这相当于幼年种群。2)更新 优势火前劣势型 即更新代数量最多, 火前代数量最 少或不存在。这类又可再分为 2 小类,第一类其 3 个世代都存在, 并且更新代的数量最多, 火后代次 之, 火前代最少; 第二类火前代不存在, 更新代数量 大于火后代。3)更新优势火后劣势型 :即更新代数 量最多, 火后代的数量最少或不存在。这类又可再 分为 2 小类,第一类其 3 个世代都存在, 并且以更新 代的数量最多, 火前代次之, 火后代最少; 第二类火 后代不存在,更新代数量大于火前代。

火后优势型指火后代存在并且数量最多。根据 更新代与火前代的存在与否及其数量关系可分为 3 种亚型:1)火后独优型 :更新代与火前代不存在, 只 有火后代, 相当于中年种群。2) 火后优势火前劣势 型即以火后代数量最多, 火前代最少或不存在。这 类再分为 2 小类, 第一类其 3 个世代都存在, 并且以 火后代数量最多, 更新代次之, 火前代最少; 第二类 火前代不存在, 火后代数量大于更新代。3) 火后优
势更新劣势型 即火后代数量最多, 更新代数量最少 或不存在。这类还可再分为 2 小类,第一类其 3 个 世代都存在, 并且以火后代的数量最多, 火前代次 之，更新代最少;第二类其更新代不存在，火后代的 数量大于火前代。

火前优势型指火前代存在并且数量最多。根据 更新代与火后代的存在否及其数量关系可分为 3 种 亚型: : 1)火前独优型: 更新代与火后代不存在, 只有 火前代 相当于成熟种群。2) 火前优势火后劣势型： 即以火前代数量最多, 火后代最少或不存在。这类 可再分为 2 小类，第一类其 3 个世代都存在，并且以 火前代数量最多，更新代次之，火后代最少;第二类 其火后代不存在, 火前代数量大于更新代。3) 火前 优势更新劣势型 即火前代数量最多, 更新代数量最 少或不存在。这类也可再分为 2 小类,第一类其 3 个世代都存在, 并且以火前代数量最多, 火后代次 之，更新代最少;第二类其更新代不存在，火前代的 数量大于火后代。

\section{2 .2 白桦种群的世代结构及其空间分布}

从景观整体上说，白哔种群的火前代、火后代和 更新代的数量分别为 40、855 和 364 株 $\mathrm{hm}^{-2}$, 其世 代结构为以火后代占优势的左偏正态分布。

从世代结构类型来说，白桦种群的世代结构类 型丰富多样，总共有 3 大类、5 亚类、11 小类。

从空间分布来看,白桦种群的世代结构在空间 上呈斑块状镶嵌分布, 空间异质性较低, 蔓延性较 大。但是, 往往存在几个面积较大、分布较集中的主 要亚型。其中, 以火后优势火前劣势型最大, 面积为 $170.42 \mathrm{hm}^{2}$, 占景观的 $31.41 \%$ 。这一种类型较为集 中地分布在景观的中部广大区域，同时在 218 林班 内沿河岸往下呈带状延伸。更新优势火前劣势型次 之, 面积为 $146.31 \mathrm{hm}^{2}$, 占景观的 $26.97 \%$ 。这一类 主要分布在 218 中下部。火后优势更新劣势型也占 有一定面积 (119.24 hm²，21.98\%）较为集中地分布 在 224 林班下部。其它各亚类的面积较小,分布较 零碎。

\subsection{3 白桦种群世代结构与火干扰的关系}

采用方差分析与多重比较, 寻求各世代相对值 在影响因子各水平间的变化情况。综合 3 个世代的 相对值的变化即为种群世代结构的变化情况。火因 子采用火干扰频率 (火干扰次数) 与平均火干扰强度 两个单因子以及两者的综合因子。

\subsubsection{1 火干扰频率对世代结构的影响}

方差分析表明，不同火频类型间白桦种群的各 
个世代的相对值都有显著差异。由表 4 可见, 更新 代与火前代的相对值都随火频表现为: 低频类 $(13.78 \%, 0.14 \%)<$ 中频类 $(36.35 \%, 2.25 \%)<$ 高 频类 $(38.64 \%, 10.21 \%)$ 。其中，低频类的更新代相 对值分别和另外两类有显著差异, 高频类的火前代 相对值分别和另外两类有显著差异。相反, 火后代 的相对值却随火频表现为: 低频类 $(86.08 \%)>$ 中频 类 $(60.09 \%)>$ 高频类 $(51.67 \%)$, 低频类与另两类 差异显著。

可见(表 4)，尽管各世代相对值对比关系发生 上述的数量变化, 但各火频类型之间世代结构没有 变化,一直保持为火后优势火前劣势型。

\subsubsection{2 火干扰强度对世代结构的影响}

方差分析表明，不同火强类型间白桦种群各世 代的相对值都存在显著差异。由表 5 可见, 白桦种 群的更新代与火前代的相对值都随火强类型表现 为:低强类 $(41.52 \%, 5.48 \%)>$ 中强类 $(27.18 \%$ ， $3.37 \%)>$ 高强类 $(18.99 \%, 0 \%)$ 。其中，低强类的 更新代与高强类有显著差异。相反, 火后代相对值 的大小顺序正好相反，表现为：低强类 $(51.25 \%)<$
中强类 $(69.72 \%)<$ 高强类 $(81.01 \%)$ 。其中，低强 类与高强类之间有显著差异。

可见(表 5)，不同火强类型之间，尽管各世代相 对值对比关系发生上述的数量变化，但世代结构没 有变化,一直保持为火后优势火前劣势型, 仅在高强 火烧地上,火前代不存在。

\subsubsection{3 火干扰频率与火干扰强度的综合影响}

方差分析表明, 不同火干扰类型间白榫种群各 世代的相对值都存在显著差异。由表 6 可见，无论 哪种火干扰类型, 劣势世代都为火前代 除了低频低 强与高频低强类的世代结构为更新优势型外, 其它 各类的世代结构皆为火后优势型。

\subsection{4 世代结构与立地条件的关系}

经数量模型 III 处理, 发现在林型组、海拨、坡 向、坡位和坡度等 5 个立地因子中，只有林型组与坡 向对世代结构的影响显著。

\subsubsection{1 林型组对世代结构的影响}

方差分析表明，不同林型组之间，白桦种群更新 代与火后代都存在显著差异, 火前代相对值差异不 显著。由表7可见，白桦种群更新代的相对值以杜

表 4 不同火频类型的白桦种群的世代结构类型与各世代的相对值 $(\%)$

Table 4 Sub-group of cohort structure and relative value $(\%)$ of cohort of Betula platyphylla population among different types of fire disturbance frequency

\begin{tabular}{|c|c|c|c|c|}
\hline $\begin{array}{c}\text { 火频类型 } \\
\text { Type of fire frequency }\end{array}$ & $\begin{array}{c}\text { 世代结构 } \\
\text { Cohort structure }\end{array}$ & $\begin{array}{c}\text { 更新代 } \\
\text { Cohort of regeneration }\end{array}$ & $\begin{array}{c}\text { 火后代 } \\
\text { Cohort of post-fire }\end{array}$ & $\begin{array}{c}\text { 火前代 } \\
\text { Cohort of pre-cohort }\end{array}$ \\
\hline $\begin{array}{c}\text { 低频类 } \\
\text { Low frequency }\end{array}$ & $\begin{array}{l}\text { 火后优势火前劣势型 Sub-groups of post-fire } \\
\text { cohort dominance \& pre-fire cohort inferiority }\end{array}$ & $13.78^{\mathrm{A}}$ & $86.08^{\mathrm{C}}$ & $0.14^{\mathrm{E}}$ \\
\hline $\begin{array}{c}\text { 中频类 } \\
\text { Middle frequency }\end{array}$ & $\begin{array}{l}\text { 火后优势火前劣势型 Sub-groups of post-fire } \\
\text { cohort dominance \& pre-fire cohort inferiority }\end{array}$ & $36.35^{\mathrm{B}}$ & $60.09^{\mathrm{D}}$ & $2.25^{\mathrm{E}}$ \\
\hline $\begin{array}{c}\text { 高频类 } \\
\text { High frequency }\end{array}$ & $\begin{array}{l}\text { 火后优势火前劣势型 Sub-groups of post-fire } \\
\text { cohort dominance \& pre-fire cohort inferiority }\end{array}$ & $38.64^{\mathrm{B}}$ & $51.67^{\mathrm{D}}$ & $10.21^{\mathrm{F}}$ \\
\hline
\end{tabular}

表中字母表示不同火干扰类型之间多重比较结果。两两之间若有一个字母相同则表示差异不显著 $(p>95 \%)$ The letters represent the results from multiple comparison between different fire types. The same letters show the insignificant difference between two types $(p>95 \%)$

表 5 不同火强类型的白桦种群的世代结构类型与各世代的相对值 $(\%)$

Table 5 Sub-group of cohort structure and relative value (\%) of cohort of Betula platyphylla population among different types of fire disturbance severity

\begin{tabular}{|c|c|c|c|c|}
\hline $\begin{array}{c}\text { 火烧强度 } \\
\text { Type of fire severity }\end{array}$ & $\begin{array}{c}\text { 世代结构 } \\
\text { Cohort structure }\end{array}$ & $\begin{array}{l}\text { 更新代 } \\
\text { Cohort of regeneration }\end{array}$ & $\begin{array}{c}\text { 火后代 } \\
\text { Cohort of post-fire }\end{array}$ & $\begin{array}{c}\text { 火前代 } \\
\text { Cohort of pre-cohort }\end{array}$ \\
\hline $\begin{array}{c}\text { 低强类 } \\
\text { Low severity }\end{array}$ & $\begin{array}{l}\text { 火后优势火前劣势型 Sub-groups of post-fire } \\
\text { cohort dominance \& pre-fire cohort inferiority }\end{array}$ & $41.52^{\mathrm{A}}$ & $51.25^{\mathrm{C}}$ & 5.48 \\
\hline $\begin{array}{c}\text { 中强类 } \\
\text { Middle severity }\end{array}$ & $\begin{array}{l}\text { 火后优势火前劣势型 Sub-groups of post-fire } \\
\text { cohort dominance \& pre-fire cohort inferiority }\end{array}$ & $27.18^{\mathrm{AB}}$ & $69.72^{\mathrm{CD}}$ & 3.37 \\
\hline $\begin{array}{l}\text { 高强类 } \\
\text { High severity }\end{array}$ & $\begin{array}{l}\text { 火后优势火前劣势型 Sub-groups of post-fire } \\
\text { cohort dominance \& pre-fire cohort inferiority }\end{array}$ & $18.99^{\mathrm{B}}$ & $81.01^{\mathrm{D}}$ & 0.00 \\
\hline
\end{tabular}

表中字母表示不同火干扰类型之间多重比较结果。两两之间若有一个字母相同则表示差异不显著 $(p>95 \%)$ The letters represent the results from multiple comparison between different fire types. The same letters show the insignificant difference between two types $(p>95 \%)$ 
表 6 不同火干扰类型的白桦种群的世代结构类型与各世代相对值 $(\%)$

Table 6 Sub-group of cohort structure and relative value (\%) of cohort of Betula platyphylla population among different types of fire disturbance

\begin{tabular}{|c|c|c|c|c|}
\hline $\begin{array}{c}\text { 火干扰类型 } \\
\text { Type of fire disturbance }\end{array}$ & $\begin{array}{c}\text { 世代结构 } \\
\text { Cohort structure }\end{array}$ & $\begin{array}{c}\text { 更新代 } \\
\text { Cohort of regeneration }\end{array}$ & $\begin{array}{c}\text { 火后代 } \\
\text { Cohort of post-fire }\end{array}$ & $\begin{array}{c}\text { 火前代 } \\
\text { Cohort of pre-cohort }\end{array}$ \\
\hline $\begin{array}{l}\text { 低频低强类 } \\
\text { Low frequency \& low severity }\end{array}$ & $\begin{array}{l}\text { 更新优势火前劣势 Sub-groups of regeneration } \\
\text { cohort dominance \& pre-fire cohort inferiority }\end{array}$ & $57.00^{*}$ & $39.30^{*}$ & $3.60^{*}$ \\
\hline $\begin{array}{l}\text { 低频中强类 } \\
\text { Low frequency \& middle severity }\end{array}$ & $\begin{array}{l}\text { 火后优势火前劣势 Sub-groups of post-fire cohort } \\
\text { dominance \& pre-fire cohort inferiority }\end{array}$ & $12.18^{\mathrm{A}}$ & $87.82^{\mathrm{A}}$ & $0.00^{A}$ \\
\hline $\begin{array}{l}\text { 低频高强类 } \\
\text { Low frequency \& high severity }\end{array}$ & $\begin{array}{l}\text { 火后优势火前劣势 Sub-groups of post-fire cohort } \\
\text { dominance \& pre-fire cohort inferiority }\end{array}$ & $11.77^{\mathrm{A}}$ & $88.23^{\mathrm{A}}$ & $0.00^{\mathrm{A}}$ \\
\hline $\begin{array}{l}\text { 中频低强类 } \\
\text { Middle frequency \& low severity }\end{array}$ & $\begin{array}{l}\text { 火后优势火前劣势 Sub-groups of pre-fire cohort } \\
\text { dominance \& post-fire cohort inferiority }\end{array}$ & $36.33^{\mathrm{BC}}$ & $57.57^{\mathrm{BC}}$ & $3.15^{\mathrm{AB}}$ \\
\hline $\begin{array}{l}\text { 中频中强类 } \\
\text { Middle frequency \& middle severity }\end{array}$ & $\begin{array}{l}\text { 火后优势火前劣势 Sub-groups of post-fire cohort } \\
\text { dominance \& pre-fire cohort inferiority }\end{array}$ & $43.84^{\mathrm{BC}}$ & $53.79^{\mathrm{BC}}$ & $2.59^{\mathrm{AB}}$ \\
\hline $\begin{array}{l}\text { 中频高强类 } \\
\text { Middle frequency \& high severity }\end{array}$ & $\begin{array}{l}\text { 火后优势火前劣势 Sub-groups of post-fire cohort } \\
\text { dominance \& pre-fire cohort inferiority }\end{array}$ & $26.86^{\mathrm{AB}}$ & $73.14^{\mathrm{AB}}$ & $0.00^{\mathrm{A}}$ \\
\hline $\begin{array}{l}\text { 高频低强类 } \\
\text { High frequency \& low severity }\end{array}$ & $\begin{array}{l}\text { 更新优势火前劣势 Sub-groups of regeneration } \\
\text { cohort dominance \& pre-fire cohort inferiority }\end{array}$ & $49.75^{\mathrm{C}}$ & $40.66^{\mathrm{C}}$ & $9.93^{\mathrm{B}}$ \\
\hline $\begin{array}{l}\text { 高频中强类 } \\
\text { High frequency \& middle severity }\end{array}$ & $\begin{array}{l}\text { 火后优势火前劣势 Sub-groups of post-fire cohort } \\
\text { dominance \& pre-fire cohort inferiority }\end{array}$ & $19.60^{\mathrm{AB}}$ & $70.56^{\mathrm{ABC}}$ & $10.70^{\mathrm{A}}$ \\
\hline
\end{tabular}

表中字母表示不同火干扰类型之间多重比较结果。两两之间若有一个字母相同则表示差异不显著 $(p>95 \%)$ The letters represent the results from multiple comparison between different fire types. The same letters show the insignificant difference between two types $(p>95 \%) \quad *$ : 表示样本数 只有 1 个 未进行多重比较 Represent no comparison because of only one sample

表 7 不同林型组的白桦种群的世代结构与各世代的相对值 $(\%)$

Table 7 Sub-group of cohort structure and relative value $(\%)$ of cohort of Betula platyphylla population among different groups of forest type

\begin{tabular}{|c|c|c|c|c|}
\hline $\begin{array}{c}\text { 林型组 } \\
\text { Groups of forest type }\end{array}$ & $\begin{array}{c}\text { 世代结构 } \\
\text { Cohort structure }\end{array}$ & $\begin{array}{c}\text { 更新代 } \\
\text { Cohort of regeneration }\end{array}$ & $\begin{array}{c}\text { 火后代 } \\
\text { Cohort of post-fire }\end{array}$ & $\begin{array}{c}\text { 火前代 } \\
\text { Cohort of pre-cohort }\end{array}$ \\
\hline 溪旁林组 Group near streams & $\begin{array}{l}\text { 火后优势火前劣势 Sub-groups of post-fire cohort } \\
\text { dominance \& pre-fire cohort inferiority }\end{array}$ & $42.23^{\mathrm{AB}}$ & $49.45^{\mathrm{CD}}$ & 8.32 \\
\hline $\begin{array}{l}\text { 赤杨林组 Group of Alnus mand- } \\
\text { shurica }\end{array}$ & $\begin{array}{l}\text { 火后优势火前劣势 Sub-groups of post-fire cohort } \\
\text { dominance \& pre-fire cohort inferiority }\end{array}$ & $48.63^{\mathrm{A}}$ & $51.22^{\mathrm{C}}$ & 0.40 \\
\hline 杜香林组 Group of Ledum palustre & $\begin{array}{l}\text { 火后优势火前劣势 Sub-groups of post-fire cohort } \\
\text { dominance \& pre-fire cohort inferiority }\end{array}$ & $23.03^{\mathrm{B}}$ & $71.91^{\mathrm{D}}$ & 4.13 \\
\hline
\end{tabular}

表中字母表示不同火干扰类型之间多重比较结果。两两之间若有一个字母相同则表示差异不显著 $(p>95 \%)$ The letters represent the results from multiple comparison between different fire types. The same letters show the insignificant difference between two types $(p>95 \%)$

香林组最大 $(48.63 \%)$ ，溪旁林组次之 $(42.23 \%)$,赤 杨林组最小 $(23.03 \%)$;杜香林组和赤杨林组间存在 显著差异。火后代相对值以赤杨林组最大 $(71.91 \%)$ 杜香林组次之 $(51.22 \%)$, 溪旁林组最小 (49.45\%) 杜香林组和赤杨林组之间存在显著差 异。

可见，不同林型组间，尽管各世代对比关系发生 上述数量变化, 但世代结构保持为火后优势火前劣 势型。

\subsubsection{2 坡向对世代结构的影响}

方差分析表明，不同坡向间白桦种群更新代和 火后代相对值存在显著差异, 火前代相对值差异不 显著。由表 8 可见, 白桦种群更新代相对值以偏东 坡较小,如东南坡( $34.28 \%)$ 、东坡 $(15.82 \%)$ 、东北 坡 (15.95\%); 以偏西坡和北坡较大，如北坡 $(49.40 \%)$ 、西坡 $(77.80 \%)$ 和西北坡 $(55.63 \%)$; 其 中，东坡分别与北坡和西北坡有显著差异，东北坡分 别和北坡和西北坡有显著差异。火后代相对值刚好 相反, 以偏东坡较大,如东南坡 $(65.72 \%)$ 、东坡 $(81.43 \%)$ 、东北坡 $(80.53 \%)$; 似偏西坡和北坡较大， 
如北坡 (44. 44\%)、西坡 (22. 20\%) 和西北坡 $(37.95 \%)$ 其中 东坡和东北坡分别与北坡和西北坡 有显著差异。火前代相对值以相对偏阳坡较小, 如东 南坡 $(0)$ 、东坡 $(0.53 \%)$ 和西坡 $(0)$; 偏阴坡较大 , 如东 北坡 $(3.68 \%)$ 、北坡 $(6.16 \%)$ 和西北坡 $(6.97 \%)$ 。

不同坡向间白桦种群的世代结构类型有两大
类,偏东坡(包括东南坡、东坡和东北坡)的世代结构 为火后优势火前劣势型 北坡与偏西坡(包括西坡和 西北坡)为更新优势火前劣势型。

\subsubsection{3 林班对世代结构的影响}

在 224 林班, 白桦种群的世代结构主要为火后 优势型，218 林班却为更新优势型。

表 8 不同坡向的白桦种群的世代结构与各世代的相对值 (\%)

Table 8 Sub-group of cohort structure and relative value $(\%)$ of cohort of Betula platyphylla population between different groups of forest type

\begin{tabular}{|c|c|c|c|c|}
\hline $\begin{array}{l}\text { 坡向 } \\
\text { Aspect }\end{array}$ & $\begin{array}{c}\text { 世代结构 } \\
\text { Cohort structure }\end{array}$ & $\begin{array}{c}\text { 更新代 } \\
\text { Cohort of regeneration }\end{array}$ & $\begin{array}{c}\text { 火后代 } \\
\text { Cohort of post-fire }\end{array}$ & $\begin{array}{c}\text { 火前代 } \\
\text { Cohort of pre-cohort }\end{array}$ \\
\hline $\begin{array}{c}\text { 东南坡 } \\
\text { Southeastern slope }\end{array}$ & $\begin{array}{l}\text { 火后优势火前劣势 Sub-groups of post-fire co- } \\
\text { hort dominance \& pre-fire cohort inferiority }\end{array}$ & $34.28^{\mathrm{ABC}}$ & $65.72^{\mathrm{DEF}}$ & 0.00 \\
\hline $\begin{array}{c}\text { 东坡 } \\
\text { Eastern slope }\end{array}$ & $\begin{array}{l}\text { 火后优势火前劣势 Sub-groups of post-fire co- } \\
\text { hort dominance \& pre-fire cohort inferiority }\end{array}$ & $15.82^{\mathrm{B}}$ & $81.43^{\mathrm{D}}$ & 0.53 \\
\hline $\begin{array}{c}\text { 东北坡 } \\
\text { Northeastern slope }\end{array}$ & $\begin{array}{l}\text { 火后优势火前劣势 Sub-groups of post-fire co- } \\
\text { hort dominance \& pre-fire cohort inferiority }\end{array}$ & $15.95^{\mathrm{BC}}$ & $80.53^{\mathrm{D}}$ & 3.68 \\
\hline $\begin{array}{l}\text { 北坡 } \\
\text { Northern slope }\end{array}$ & $\begin{array}{l}\text { 更新优势火前劣势型 Sub-groups of regenera- } \\
\text { tion cohort dominance \& pre-fire cohort inferiority }\end{array}$ & $49.40^{\mathrm{A}}$ & $44.44^{\mathrm{EG}}$ & 6.16 \\
\hline $\begin{array}{c}\text { 西坡 } \\
\text { Western slope }\end{array}$ & $\begin{array}{l}\text { 更新优势火前劣势型 Sub-groups of regenera- } \\
\text { tion cohort dominance \& pre-fire cohort inferiority }\end{array}$ & $77.80^{\mathrm{AB}}$ & $22.20^{\mathrm{DG}}$ & 0.00 \\
\hline $\begin{array}{l}\text { 西北坡 } \\
\text { Northwestern slope }\end{array}$ & $\begin{array}{l}\text { 更新优势火前劣势型 Sub-groups of regenera- } \\
\text { tion cohort dominance \& pre-fire cohort inferiority }\end{array}$ & $55.63^{\mathrm{A}}$ & $37.95^{\mathrm{FG}}$ & 6.97 \\
\hline
\end{tabular}

表中字母表示不同火干扰类型之间多重比较结果。两两之间若有一个字母相同则表示差异不显著 $(p>95 \%)$ The letters represent the results from multiple comparison between different fire types. The same letters show the insignificant difference between two types $(p>95 \%)$

\section{4 讨论与结论}

\section{1 基于世代数的世代结构}

大兴安岭北部原始林因为频繁的火干扰,造成 白桦种群世代数较丰富, 世代结构在空间上呈斑块 状镶嵌分布, 瞢延性较高, 空间异质性较低。在多样 化的世代结构类型中, 以一代型与二代型这两种世 代结构占据主要地位, 这些主要世代结构在空间上 的分布相对较聚集, 连接性较好, 往往以一到两个主 斑块及众多零星小玟块构成。其它类型的世代结构 的面积较小, 空间分布较破碎。主要原因可能是频 繁的火干扰及其空间分布决定的(徐化成等，1997; 邱扬等, 2003)。

白桦种群的世代数受火干扰频率、火干扰强度 及两者的综合影响。低频或高强度的火干扰易造成 种群的世代数减少, 世代结构趋于简单; 高频或低强 度的火干扰易造成种群的世代数增多,世代结构趋 于复杂。相对来说以火干扰频率更为显著。白桦种 群世代数随着火烧次数增多而增加，低频类都为一
代型、中频类为二代型、高频类为三代型 相反，火频 相同火强增高, 世代数变化不显著, 世代结构类型基 本一致。

白桦种群的世代数还随林型组类型而变化, 以 溪旁林组 $>$ 赤杨林组 $>$ 杜香林组。造成这种现象的 主要原因是不同林型组之间干扰状况的差别(徐化 成等，1997)。尽管溪旁林组的火干扰次数较少，但 是其极易成 醉林”, 风倒干扰严重 ;雨季大水不定期 地冲刷下层植被, 露出矿质土, 有利幼苗的发生; 另 外该林型组的生产力较高, 也是造成该林型组的世 代数多的一个原因。相对于杜香林组来说, 赤杨林 组的火干扰次数较多, 生产力较高, 因而世代数相对 较多(邱扬等, 2003)。

\section{2 基于火干扰的世代结构}

从总体来说，白桦种群的世代结构是火后优势 火前劣势型的倒 J”形, 反应了该种群的抗火性较 差, 但是火后恢复性很强。可见，在火频繁的大兴安 岭原始林景观中, 白桦种群表现为 $r$ 选择的特征, 依 靠火后恢复性维持景观中的地位(邱扬等，1998)。 
白桦种群的世代结构类型较丰富，存在 3 大类、 5 亚类、11 小类,空间分布呈斑块状的镶嵌体, 空间 异质性较低, 蔓延性较大。这是由景观上火干扰的 多样性所控制的（徐化成等，1997)，也反映了该种 群对火干扰适应机制的多样性(丘扬等，1998)。在 多样化的世代结构类型中，以火后优势火前劣势型 和更新优势火前劣势型这两个主要类型的面积较 大, 空间分布较聚集。造成这种空间分布主要原因 可能是因为最近一次火干扰强度的空间变异 ${ }^{1)}$ 。

虽然白桦种群的各世代受到火干扰频率、火干 扰强度及两者的综合作用影响, 但是对世代结构的 影响不明显。随火频的增高或火强的降低, 更新代 和火前代的比例增大, 火后代的优势性降低, 优势世 代从火后代向更新代转变, 但劣势世代保持为火前 代不变。在中高强火烧地上, 白桦种群的世代结构 为火后优势火前劣势型, 表明种群以火后恢复性为 主要对策维持稳定性, 呈现出较强的进展性。随火 频的增高或火强的降低，火后恢复性所占的地位显 著降低, 自我恢复性与抗火性则显著增强。在低强 火烧地上 种群世代结构可能已成为更新优势火前 劣势型, 主要以自我恢复性及一定的火后恢复性这 两种对策维持在景观中的地位(丘扬等，1998)。

白桦种群的世代结构还随立地条件而变化。不 同林型组之间, 白桦种群的优势世代(火后代和更新 代)存在显著的数量差异, 但是世代结构不变, 保持 为火后优势火前劣势型。坡向之间白桦种群的世代 结构主要有两种: 偏东坡为火后优势火前劣势型, 偏 西及北坡为更新优势火前劣势型。坡向之间的主要 区别在于优势世代所占的比重的变化。这是因为偏 东坡比偏西坡和北坡的火干扰强度较大、火干扰频 率较小的缘故 ${ }^{1)}$ 。林班之间, 白桦种群的世代结构 也主要有两种,224 林班为火后优势型,218 林班却 为更新优势型。这可能是因为林班的坡向差异造成 的火干扰状况与立地条件的差异。218 林班偏西而 224 林班偏东, 218 林班比 224 林班要干燥些, 其火 干扰频率大些, 而火干扰强度要小一些(徐化成等, 1997)。

\section{参 考 文 献}

Fan ZF (范兆飞), Xu HC (徐化成), Yu RY (于汝元)

(1991). Study on the relation among the diameter, height distribution and age structure of Larix gemelini. Journal of Beijing
Forestry University (北京林业大学学报), 12 (Suppl. ), 75 80. (in Chinese)

Fan ZF (范兆飞), Xu HC (徐化成), Yu RY (于汝元) (1992). A study on the species group age structure of Larix gmelinii population and its relation to disturbance in the north Daxinganling Mountains. Scientia Silvae Sinicae (林业科学), 28, 2 - 11. (in Chinese with English abstract)

Glin-Leven DC, Peet RK, Veblin TT (1992). Plant Succession: Theory and Prediction. Chapman \& Hall, New York.

Gu YC (顾云春) (1985). The succession of forest communities in the forest region of the Greater Xingan Mountains. Acta Phytoecologica et Geobotanica Sinica (植物生态学与地植物学丛刊), 9, 64 - 70. (in Chinese with English abstract)

Meng XY (孟宪宇) (1989). Analyses for structures of age in natural Larix gmelinii stands. Journal of Beijing Forestry University (北京林业大学学报), 11 (3), 17-23. (in Chinese with English abstract)

Oliver CD, Larson BC (1990). Forest Stand Dynamics. McGrawHill, New York.

Pickett STA, White PS (1985). The Ecology of Natural Disturbance and Patch Dynamics. Academic Press, New York.

Qiu Y (邱扬), Du JL (杜建林) (1997). Age structure survey of tree population in forest ecology studies. Journal of Shanxi University (Natural Science Edition) (山西大学学报 (自然科学 版)), 20, 107 - 110. (in Chinese with English abstract)

Qiu Y (邱扬), Li ZD (李湛东), Xu HC (徐化成) (1997).

Study on the relation between the stability of Larix gemelini population and the fire disturbance. Bulletin of Botanical Research (植物研究), 17, 441 - 446. (in Chinese with English abstract)

Qiu Y (邱扬), Li ZD (李湛东), Zhang YJ (张玉钧), Xu HC (徐化成), Yu RY (于汝元) (2003). Study on the cohort structure of Larix gemelinii population in virgin forest in northern of Daxing' an Mountains. Scientia Silvae Sinicae (林业科学), 39(3), 15-22. (in Chinese with English abstract) Qiu Y (邱扬), Li ZD (李湛东), Yu RY (于汝元) (1998). Study on the relation between the stability of Betula platyphylla population and the fire disturbance. Bulletin of Botanical Research (植物研究), 18, $321-327$. (in Chinese with English abstract)

Shan JP (单建平), Xu ZB (徐振邦), Tao DL（陶大立） (1990). Fire and the seed germination of Larix gmelinii. Scientia Silvae Sinicae (林业科学), 26, 545 - 549. (in Chinese with English abstract)

Sun XY (孙晓玉), Lu ZH (陆兆华), Yu XJ (于兴军), Sang WG (桑卫国) (2005). Age structure dynamics of Eupatorium adenophorum populations and its implications for control. Acta Phytoecologica Sinica（植物生态学报), 29, 373-379. (in 
Chinese with English abstract)

Xu HC (徐化成), Fan ZF (范兆飞), Yu RY (于汝元) (1993).

Age structure dynamics of virgin Larix gmelinii forest. Chinese Journal of Applied Edology (应用生态学报) , 4, 229-233. (in Chinese with English abstract)

Xu HC (徐化成), Li ZD (李湛东), Qiu Y (兵扬) (1997). Fire disturbance history in virgin forest in northern region of Daxinganling Mountains. Acta Ecologica Sinica (生态学报), 17, 337 343. (in Chinese with English abstract)

Yang YH (杨允菲), Zheng HY (郑慧莹), Li JD (李建东) (2001). The effects of grazing on age structure in clonal popula- tions of Agropyron michnoi. Acta Phytoecologica Sinica (植物生 态学报), 25, 71-75. (in Chinese with English abstract)

Zhao HX (赵惠勋), Wang YH (王义弘), Li JQ (李俊清), He XL (何晓兰) (1987). The age structure, spatial pattern and management of natural forest of Larix gemelini, Tahe forestry bureau. Journal of Northeast Forestry University (东北林业大学学 报), 15(Suppl.), 60-64. (in Chinese)

Zheng HL (郑焕能), Jia SQ (贾松清), Hu HQ (胡海清) (1986) . Forest fire and forest rehabilitation in the Daxing' anling Mountains. Journal of Northeast Forestry University (东北林业大 学学报), 14, 1-7. (in Chinese with English abstract)

责任编委 : 马克平 责任编辑 :刘丽娟 